\title{
Feasibility and Accuracy of Transduodenal Endoscopic Ultrasound- Guided Fine-Needle Aspiration of Solid Lesions Using a 19-Gauge Flexible Needle: A Multicenter Study
}

\author{
Germana de Nucci ${ }^{1}$, Maria Chiara Petrone ${ }^{2}$, Nicola Imperatore ${ }^{3,4}$, Edoardo Forti $^{5}$, Roberto Grassia ${ }^{6}$, Silvia Giovanelli ${ }^{7}$, Laura \\ Ottaviani ${ }^{8}$, Vincenzo Mirante ${ }^{9}$, Giuseppe Sabatino ${ }^{10}$, Carlo Fabbri ${ }^{7}$, Mauro Manno ${ }^{9}$, Paolo Giorgio Arcidiacono ${ }^{2}$ and Gianpiero \\ Manes $^{1}$ \\ ${ }^{1}$ Gastroenterology and Endoscopy Unit, ASST Rhodense, Garbagnate Milanese, Milan, ${ }^{2}$ Bilio Pancreatic Endoscopy and Endoscopy \\ Ultrasound Unit, San Raffaele Hospital, Milan, ${ }^{3}$ Gastroenterology Unit, Federico II University, Naples, ${ }^{4}$ Gastroenterology and \\ Endoscopy Unit, AORN Antonio Cardarelli, Naples, ${ }^{5}$ Endoscopy Unit, Ca Grande Niguarda Hospital, Milan, ${ }^{6}$ Endoscopy Unit, Cremona \\ Hospital, Cremona, ${ }^{7}$ Gastroenterology and Endoscopy Unit, Forli-Cesena Hospital, Forlì, ${ }^{8}$ Gastroenterology Unit, Santa Maria Hospital, \\ Perugia, ${ }^{9}$ Endoscopy Unit, Carpi Civil Hospital, Carpi, ${ }^{10}$ Gastroenterology Unit, Cosenza Civil Hospital, Cosenza, Italy
}

Background/Aims: Endoscopic ultrasound-guided fine-needle aspiration (EUS-FNA) is the go-to method for obtaining samples from gastrointestinal tract and pancreatic lesions. When the transduodenal approach is utilized, the use of a more flexible needle, such as a nitinol 19-gauge $(G)$ needle, has been recommended. The aim of this study was to evaluate the feasibility and accuracy of 19-G flexible aspiration needles in obtaining samples from solid lesions through a transduodenal approach.

Methods: This was a retrospective analysis of prospectively collected data from eight Italian endoscopy centers. Consecutive patients with solid lesions who underwent transduodenal EUS-FNA with a 19-G flexible needle were included.

Results: A total of 201 patients were enrolled. According to histology, EUS, radiology and 12 months of follow-up, 151 patients had malignant lesions and 50 patients had benign lesions. EUS-FNA was feasible in all cases. An adequate histologic sample was obtained in all except eight cases (96.1\%). The sensitivity of EUS-FNA was $92.1 \%$ (95\% confidence interval [CI], 86.8\%-95.7\%), and the specificity was $100 \%$ (95\% CI, $90.5 \%-100 \%)$. The positive predictive value was $100 \%$ (95\% CI, $93.4 \%-100 \%)$, and the negative predictive value was $74 \%$ (95\% CI, 62.8\%-82.7\%). The diagnostic accuracy was $93.5 \%$ (95\% CI, 89.2\%-96.5\%).

Conclusions: The transduodenal approach for obtaining samples from solid lesions using a 19-G flexible needle seems feasible and accurate. Clin Endosc 2021;54:229-235

Keywords: Endoscopic ultrasound-guided fine-needle aspiration; Endosonography; Feasibility studies

\section{INTRODUCTION}

Endoscopic ultrasound-guided fine-needle aspiration (EUS-FNA) is a safe, simple, and accurate method for cor-

Received: February 24, 2020 Revised: April 1, 2020

Accepted: April 1, 2020

Correspondence: Germana de Nucci

Gastroenterology and Endoscopy Unit, ASST Rhodense, Garbagnate Milanese, Viale Carlo Forlanini 95, Garbagnate Milanese, Milan 20024, Italy

Tel: +39-02-994302533, Fax: +39-02-994302905, E-mail: germanadenucci1@ gmail.com

ORCID: https://orcid.org/0000-0001-8758-654X

(c) This is an Open Access article distributed under the terms of the Creative Commons Attribution Non-Commercial License (http://creativecommons.org/ licenses/by-nc/3.0) which permits unrestricted non-commercial use, distribution, and reproduction in any medium, provided the original work is properly cited. rectly diagnosing pancreatic lesions, gastrointestinal (GI) submucosal lesions, and abdominal and thoracic lymph node metastases. When performed by trained cytopathologists, its results are reliable, ${ }^{1}$ with a diagnostic yield of approximately of $90 \%-95 \%$ and an overall sensitivity and specificity of $90 \%$ and $100 \%$, respectively. ${ }^{2}$ However, the diagnostic sensitivity is high only when rapid on-site evaluation (ROSE) of specimens is performed to assess their adequacy. ${ }^{3,4}$ Most institutions do not have skilled pathologists available to perform ROSE. Moreover, EUS-FNA may not be totally effective because it provides cells that have been largely disrupted from their original arrangement and thus difficult to interpret. ${ }^{5}$ Meanwhile, fine-needle biopsy (FNB) provides core tissue with better preservation of the cellular architecture and is likely to offer 
better diagnostic accuracy because it can also obtain tissue for ancillary techniques. FNB may be specifically requested by pathologists to establish a definitive diagnosis in challenging cases, to fully characterize certain neoplasms (such as lymphomas and GI stromal tumors), ${ }^{6}$ or when FNA is inconclusive, which requires a new sampling procedure, resulting in delayed treatments and possible morbidity as well as additional costs. ${ }^{7}$ Performing at least three EUS-FNB passes using a 19-gauge (G) needle or any core biopsy needle has been proposed to improve the size and quality of the tissue sample. However, such needles are stiffer and more difficult to use than other needles, and may consequently fail when biopsy is performed with the scope in a bent, torqued position. ${ }^{8,9} 19-\mathrm{G}$ needles made of a nitinol metal alloy have increased flexibility and are thus likely to guarantee easier and safer tissue sampling even in the duodenum. ${ }^{10-12}$ They have been introduced into clinical practice in recent years; however, Attili et al. ${ }^{13}$ and Laquière et al. ${ }^{14}$ have raised some doubts about the feasibility, accuracy, and safety of these needles when used to sample solid periduodenal lesions. In particular, these two studies reported an accuracy of $73 \%$ and $80 \%$, respectively, with technical and clinical failures occurring essentially in the pancreatic head or uncinate process lesions. ${ }^{13,14}$ Our study was designed to evaluate the feasibility, safety, and accuracy of 19-G nitinol flexible needles in patients with solid lesions punctured from the first, second, and third portions of the duodenum.

\section{MATERIALS AND METHODS}

\section{Study population and study design}

We performed a retrospective analysis of prospective databases in eight tertiary endoscopic centers in Italy, and included consecutive adult patients with solid lesions who underwent EUS with tissue sampling through the duodenum by using 19-G needles (Expect ${ }^{\mathrm{TM}}$ standard type [Ex-19G] or Expect $^{\mathrm{TM}}$ Slimline flexible type [ExF-19G]; Boston Scientific, Marlborough, MA, USA) between January 2015 and December 2018. Consecutive adult patients with solid lesions (pancreatic head, uncinate process, or pancreatic neck masses; periduodenal lymph node masses; biliary masses; liver masses; and periduodenal abdominal masses) who underwent transduodenal EUS-FNA with a 19-G needle were considered eligible for inclusion. Patients with an altered anatomy of the upper GI tract because of a prior surgery in the esophagus, stomach, and duodenum were excluded for safety reasons.

In all centers, EUS was performed using a linear array echoendoscope (Pentax EG-3870UTK [Pentax, Tokyo, Japan] or Olympus UCT-180 [Olympus, Tokyo, Japan]) with the patients in the left decubitus position and under conscious/ deep sedation. Tissue acquisition was performed through the duodenum with one of the two aforementioned 19-G needles.

The EUS features of lesions (size, location, vascular invasion) and the technical details of FNA (duodenal site through which the FNA was performed, number of passes, type of needle used) were recorded for each patient. The feasibility of FNA was defined as the ability of performing EUS-FNA through the duodenum by introducing and advancing the needle into the target lesion.

Tissue acquisition was performed according to a well-defined and homogeneous protocol describes as follows. Briefly, after the target lesion was endosonographically visualized and the region was scanned for vessels using color and pulsed Doppler, biopsy was performed. The needle was positioned under FNA guidance with the stylet inside and retracted a few millimeters from the needle shaft. Once the lesion was penetrated, the stylet was gently removed with a slow pull technique. FNA was performed using the fanning technique, in which puncture was started at the left margin of the tumor mass and then the needle was moved back and forth in fanlike motion until the right margin of the tumor was sampled. The number of passes was not established initially, but independently decided by each endoscopist according to the size and features of the specimens obtained. The patients were monitored for 2 hours after the procedure and then discharged from the endoscopy unit. Further follow-up was performed by evaluating the electronic clinical records for in-hospital patients or through a phone follow-up 7 days after the procedure for outpatients.

The procedure was considered "safe" if no procedure-related complications, such as perforation, pancreatitis, infectious adverse events, bile peritonitis, malignant seeding, and hemorrhage, occurred.

Because no pathologist was present in the endoscopy room, the endoscopists recovered and stored the FNA samples for further processing, and performed a macroscopic on-site evaluation of the obtained specimen. Samples were embedded in formalin and transported within 1 hour to the pathology department. All samples were processed for histologic analysis at the pathology department of each unit. The FNA samples were evaluated by a dedicated pathologist at each hospital. Tissue sections of 3-4 $\mu \mathrm{m}$ size were stained with hematoxylin-eosin for morphologic evaluation and/or subjected to different immunohistochemical analysis methods. A histologically adequate sample was defined as an architecturally intact piece of a tissue sample from the target lesion that is deemed sufficient for histologic evaluation by the pathologist.

The reference final diagnosis was obtained from the histologic assessment of surgical specimens in operated patients. For non-operated patients, the final diagnosis was obtained by 
combining the findings of EUS and/or other radiologic techniques (computed tomography, magnetic resonance imaging) with compatible clinical/radiologic follow-up findings over at least 12 months.

\section{Statistical analysis}

Data were analyzed using the Statistical Package for Social Sciences (SPSS software version 15.0; SPSS Inc., Chicago, IL, USA) for Windows. The descriptive statistics used included mean values and standard deviation for continuous variables, and percentages and proportions for categorical variables. Statistical analysis was performed using the chi-square and Mann-Whitney $U$ tests, as appropriate. The sensitivity, specificity, positive predictive value (PPV), negative predictive value (NPV), and overall accuracy of EUS-FNA with a 19-G needle were calculated with $95 \%$ confidence intervals (CIs). Furthermore, a subanalysis of the diagnostic accuracy of each needle was also performed. Inadequate samples for histologic evaluation or technical failures were considered false-negative cases.

A binary logistic regression was used to examine the possible predictors for a correct diagnosis. Our regression model used a backward stepwise selection (Wald) method. All continuous variables were dichotomized as normal versus abnormal (yes vs. no). The coefficients obtained from the logistic regression analysis were also expressed in terms of odds ratios (ORs) of event occurrence. A $p$-value of $<0.05$ was considered statistically significant.

\section{RESULTS}

A total of 201 patients $(60.2 \%$ men, mean age $67.5 \pm 13.6$ years) finally met the inclusion criteria and were enrolled. Table 1 describes the clinical and pathologic features of the study population.

Most patients presented a pancreatic mass ( $n=143,71.1 \%)$, localized at the pancreatic head in $122(85.3 \%)$, at the uncinate process in $16(11.2 \%)$, and at the pancreatic neck in $5(3.5 \%)$ patients. Thirty-four patients (16.9\%) had enlarged and abnormal lymph nodes in the periduodenal $(n=7,20.5 \%)$, perimesenteric $(n=9,26.5 \%)$, hepatic hilar $(n=15,44.1 \%)$, and aortocaval $(n=3,8.8 \%)$ sites. Thirteen patients $(6.5 \%)$ had an extrahepatic biliary mass, whereas the remaining 11 patients (5.6\%) presented a hepatic mass. In $21.9 \%$ of the cases, the mass had EUS features consistent with vascular invasion.

The mean lesion size was $32.9 \pm 13.9 \mathrm{~mm}$. EUS-FNA was performed using the Ex-19G needle in $43.8 \%$ of the cases and ExF-19G in the remaining 56.2\%, through the duodenal bulb $(47,23.4 \%)$, second duodenal portion $(143,71.1 \%)$, and third duodenal portion (11, 5.5\%). The mean number of passes was $2.06 \pm 0.7$.

No major or minor complications occurred during or after the sampling procedure. FNA was feasible in all cases. The needle was easily inserted into the scope and withdrawn from the working channel in all 201 cases, with no registered scope damages.

An adequate histologic sample was obtained in 193 cases (96.1\%). According to histology, 149 of 193 lesions were finally classified as malignant and 44 as benign, and their different features are summarized in Table 1.

The diagnostic accuracy of EUS-FNA was $93.5 \%$ (95\% CI, $89.2 \%-96.5 \%)$, with a sensitivity of $92.1 \%$ (95\% CI, $86.8 \%-$ $95.7 \%)$, specificity of $100 \%$ (95\% CI, $90.5 \%-100 \%)$, PPV of $100 \%$, and NPV of $74 \%$ ( $95 \%$ CI, $62.8 \%-82.7 \%$ ). We found that Ex-19G and ExF-19G had similar sensitivity, specificity, and diagnostic accuracy (Table 2). However, ExF-19G was more frequently used to obtain tissue from the liver $(8.8 \%$ vs. $1.1 \%, p=0.03)$ and permitted fewer passes $(1.82 \pm 0.6$ vs. 2.38 $\pm 0.8, p=0.002$ ). By contrast, Ex-19G was more frequently used to perform FNA from the pancreatic head $(69.3 \%$ vs. $53.9 \%, p=0.02$ ) and through the third duodenal portion (11.3\% vs. $0.9 \%, p=0.001)$ (Table 1$)$.

In binary logistic regression, only vascular invasion (OR, $6.4 ; p<0.001)$ and age $>65$ years $(\mathrm{OR}, 1.81 ; p<0.01)$ were independent factors associated with a correct diagnosis using the ExF-19G needle (Table 3). Conversely, sex, site of the mass (pancreas, lymph nodes, bile ducts, or liver), size of the mass, number of passes, and site of puncture (bulb, second duodenal portion, or third duodenal portion) were not predictive of a correct diagnosis.

\section{DISCUSSION}

The present multicenter study was performed to explore the feasibility, safety, and accuracy of FNA sampling with a 19-G needle made of a stainless nitinol alloy in a large cohort of consecutive patients with solid lesions approached from the duodenum. The procedure was technically successful and safe in all patients, obtaining an adequate sample for histologic examination in $96.1 \%$ of the cases with an overall diagnostic accuracy of $93.5 \%$.

In the last 15 years, there has been an increasing interest in developing EUS tools for obtaining core samples for histologic analysis. ${ }^{15,16}$ This interest arises from several sources, mainly the necessity to reach a reliable diagnosis without ROSE, as well as to obtain a core tissue for molecular profiling and targeted therapies in GI/pancreatic cancer, with fewer passes and less time consumed. ${ }^{17}$ 
Table 1. Clinical and Pathologic Information of the Study Population

\begin{tabular}{|c|c|c|c|c|c|}
\hline & & Entire population $(n=201)$ & Ex-19G $(n=88)$ & ExF-19G $(n=113)$ & $p$-value \\
\hline Male gender, $n(\%)$ & & $121(60.2)$ & $54(61.4)$ & $67(59.3)$ & 0.7 \\
\hline Age, yr $($ mean $\pm S D)$ & & $67.5 \pm 13.6$ & $71.7 \pm 12.4$ & $64.2 \pm 13.6$ & 0.5 \\
\hline Pancreatic lesion, $n(\%)$ & Head & $122(60.7)$ & $61(69.3)$ & $61(53.9)$ & $0.02^{\mathrm{a})}$ \\
\hline & Uncinate process & $16(7.9)$ & $8(9.1)$ & $8(7.1)$ & 0.6 \\
\hline & Neck & $5(2.5)$ & $3(3.4)$ & $2(1.8)$ & 0.4 \\
\hline Lymph nodes, $n(\%)$ & Periduodenal & $7(3.4)$ & $3(3.4)$ & $4(3.5)$ & 0.9 \\
\hline & Perimesenteric & $9(4.4)$ & $3(3.4)$ & $6(5.3)$ & 0.5 \\
\hline & Hepatic hilum & $15(7.5)$ & $5(5.7)$ & $10(8.8)$ & 0.4 \\
\hline & Aortocaval & $3(1.5)$ & $0(0)$ & $3(2.6)$ & 0.3 \\
\hline Bile ducts masses, $n(\%)$ & & $13(6.5)$ & $4(4.5)$ & $9(7.9)$ & 0.3 \\
\hline Liver masses, $n(\%)$ & & $11(5.6)$ & $1(1.1)$ & $10(8.8)$ & $0.03^{\mathrm{a})}$ \\
\hline Vascular invasion, $n(\%)$ & & $44(21.9)$ & $23(26.1)$ & $21(18.6)$ & 0.2 \\
\hline Mass dimensions, $\mathrm{mm}(\mathrm{m}$ & & $32.95 \pm 13.96$ & $36.1 \pm 13.2$ & $30.5 \pm 14.1$ & 0.8 \\
\hline Number of passes (mean \pm & & $2.06 \pm 0.76$ & $2.38 \pm 0.8$ & $1.82 \pm 0.6$ & $0.002^{\mathrm{a})}$ \\
\hline FNA duodenal site, $n(\%)$ & Duodenal bulb & $47(23.4)$ & $16(18.2)$ & $31(27.4)$ & 0.1 \\
\hline & Second portion & $143(71.1)$ & $62(70.4)$ & $81(71.7)$ & 0.8 \\
\hline & Third portion & $11(5.5)$ & $10(11.3)$ & $1(0.9)$ & $0.001^{\mathrm{a})}$ \\
\hline Feasibility & & $100 \%$ & $100 \%$ & $100 \%$ & 1 \\
\hline Inadequate samples, $n(\%)$ & & $8(3.9)$ & $2(2.3)$ & $6(5.3)$ & 0.3 \\
\hline Malignant lesions in adeq & ng, $n(\%)$ & $149(74.1)$ & $69(78.4)$ & $80(70.8)$ & 0.2 \\
\hline Pancreas, 111 (74.5) & Adenocarcinoma & 87 & 35 & 52 & 0.4 \\
\hline & NET & 11 & 8 & 3 & 0.3 \\
\hline & Other metastasis & 9 & 3 & 6 & 0.1 \\
\hline & Primitive lymphoma & 4 & 1 & 3 & 0.7 \\
\hline Lymph node, 25 (16.8) & Lymphoma & 20 & 11 & 9 & 0.4 \\
\hline & Metastasis & 5 & 3 & 2 & 0.1 \\
\hline Bile ducts, 8 (5.4) & Cholangiocarcinoma & 8 & 5 & 3 & 0.1 \\
\hline Liver, 5 (3.3) & Metastasis & 5 & 3 & 2 & 0.3 \\
\hline Benign lesions in adequat & $n(\%)$ & $44(21.9)$ & $17(19.3)$ & $27(23.9)$ & 0.2 \\
\hline Pancreas, 23 (52.3) & Chronic pancreatitis & 22 & 9 & 13 & 0.6 \\
\hline & Schwannoma & 1 & 1 & 0 & 0.8 \\
\hline Lymph node, 12 (27.3) & Tuberculosis & 9 & 3 & 6 & 0.7 \\
\hline & Sarcoidosis & 3 & 1 & 2 & 0.5 \\
\hline Bile ducts, 3 (6.8) & PSC & 3 & 1 & 2 & 0.5 \\
\hline Submucosal, 6 (13.6) & Low grade GISTs & 6 & 2 & 4 & 0.4 \\
\hline
\end{tabular}

Ex-19G, Expect ${ }^{\mathrm{TM}}$ standard type; ExF-19G, Expect ${ }^{\mathrm{TM}}$ Slimline flexible type; FNA, fine-needle aspiration; GIST, gastrointestinal stromal tumor; NET, neuroendocrine tumor; PSC, primary sclerosing cholangitis; SD, standard deviation.

${ }^{\text {a) }}$ Statistically significant difference. 
Table 2. Differences between Needles

\begin{tabular}{lccc}
\hline & Ex-19G $(\boldsymbol{n}=\mathbf{1 1 3})$ & ExF-19G $(\boldsymbol{n}=\mathbf{8 8})$ & $\boldsymbol{p}$-value \\
\hline Sensitivity & $93.2 \%$ & $91.1 \%$ & 0.5 \\
Specificity & $100 \%$ & $100 \%$ & 1 \\
PPV & $100 \%$ & $100 \%$ & 1 \\
NPV & $73.6 \%$ & $74.2 \%$ & 0.3 \\
Accuracy & $94.4 \%$ & $92.9 \%$ & 0.4 \\
\hline
\end{tabular}

Ex-19G, Expect ${ }^{\mathrm{TM}}$ standard type; ExF-19G, Expect ${ }^{\mathrm{TM}}$ Slimline flexible type; NPV, negative predictive value; PPV, positive predictive value.

Table 3. Factors Associated with a Correct Diagnosis Using the Expect ${ }^{\mathrm{TM}}$ Slimline Flexible Type Needle

\begin{tabular}{|c|c|c|c|c|c|c|c|}
\hline \multirow{2}{*}{ Variables } & & \multicolumn{3}{|c|}{ Univariate analysis } & \multicolumn{3}{|c|}{ Binary logistic regression } \\
\hline & & OR & $95 \% \mathrm{CI}$ & $p$-value & OR & $95 \% \mathrm{CI}$ & $p$-value \\
\hline Male sex & & 1.10 & $0.8-1.4$ & 0.4 & & & \\
\hline Age $>65 \mathrm{yr}$ & & 1.63 & $0.9-3.6$ & $0.06^{\mathrm{a})}$ & 1.81 & $1.2-4.3$ & $<0.01^{\text {a) }}$ \\
\hline Pancreatic mass & & 1.2 & $0.8-1.5$ & 0.6 & & & \\
\hline Lymph nodes & & 1.08 & $0.7-1.6$ & 0.7 & & & \\
\hline Bile ducts mass & & 0.8 & $0.5-1.8$ & 0.8 & & & \\
\hline Hepatic mass & & 1.3 & $0.8-1.7$ & 0.5 & & & \\
\hline Mass size $>30 \mathrm{~mm}$ & & 1.12 & $0.8-1.7$ & 0.6 & & & \\
\hline Vascular invasion & & 1.5 & $1.1-1.9$ & $<0.01^{\text {a) }}$ & 6.4 & $3.4-11.6$ & $<0.001^{\mathrm{a})}$ \\
\hline \multirow[t]{3}{*}{ FNA duodenal site } & Bulb & 1.2 & $0.8-1.7$ & 0.3 & & & \\
\hline & Second portion & 1.3 & $0.7-1.9$ & 0.5 & & & \\
\hline & Third portion & 1.1 & $0.9-1.6$ & 0.6 & & & \\
\hline Number of passes $>2$ & & 1.3 & $0.7-1.8$ & 0.3 & & & \\
\hline
\end{tabular}

CI, confidence interval; FNA, fine-needle aspiration; OR, odds ratio.

${ }^{\text {a) }}$ Statistically significant difference.

In 2006, Yasuda et al. ${ }^{18}$ first described the biopsy of mediastinal or intra-abdominal lymphadenopathy using a standard $19-\mathrm{G}$ needle, with an overall diagnostic accuracy of $98 \%$ and a very high chance $(88 \%)$ of correct subtyping of lymphomas, in a cohort of 104 patients. The lesions were approached through the duodenum in only a few cases. In 2011, Larghi et al. ${ }^{19}$ conducted a prospective study using the 19-G Echo tip Cook needle in 120 patients with suspicious lesions after a first FNA sampling. FNA showed a very high feasibility (98\%) and diagnostic accuracy (93\%); however, the authors excluded lesions localized in the head or uncinate process of the pancreas to avoid the transduodenal route and its complications. More recently, Varadarajulu et al. ${ }^{20}$ and Kumbhari et al. ${ }^{21}$ described their experience with the 19-G needles from Boston Scientific, highlighting the usefulness of the needles in the safe sampling of lesions from all different GI sites independently from the scope position.

However, when using a 19-G needle, the transduodenal approach remains controversial in terms of feasibility, safety, and accuracy. The recent studies by Attili et al. ${ }^{13}$ and Laquière et al. $^{14}$ have raised some doubts about the real efficacy of Ex-19G and ExF-19G for transduodenal biopsy, for apparently discordant reasons. In particular, Attili et al. ${ }^{13}$ reported a technical success rate $>90 \%$ in five of six involved centers; however, the procurement yield was rather low, ranging from $64 \%$ to $88 \%$, with two centers presenting an unacceptable rate of $<70 \%$ (missing a correct diagnosis in one of four patients). The authors ascribed most of the technical failures to the inability of placing the scope in a proper position for puncturing the lesion; however, the registered low procurement yield likely only partly relies on the low technical success. Interestingly, in the study, the authors removed the stylet of the needle before 
introducing the needle into the scope channel with the aim of increasing the needle flexibility. This likely contributed to the occurrence of major complications in $2.4 \%$ of the patients, who all required hospitalization. We suggest, however, that this may also explain the low procurement yield registered in the study, as, without the stylet, the needle tip may have been occluded during the insertion into the target lesion, thus providing smaller and crushed samples. Meanwhile, Laquière et al. ${ }^{14}$ performed a multicenter prospective randomized study comparing the $19-\mathrm{G}$ needle with a standard $22-\mathrm{G}$ needle. The diagnostic accuracy was $69.5 \%$ and $87.3 \%$ for the $19-\mathrm{G}$ and 22-G needle, respectively, with an $8 \%$ incidence of minor postexamination complications (nine cases for the 19-G needle and nine cases for the $22 \mathrm{G}$ needle). However, the technical success of the $19-\mathrm{G}$ needle was only $86.4 \%$ (vs. $100 \%$ for the 22-G needle), which was mainly responsible for the low accuracy of the $19-\mathrm{G}$ needle.

Our study registered a technical success of $100 \%$, a result that is likely reliable owing to the homogeneity of study protocol and high flexibility of the needles. ${ }^{12}$ Similarly, the procurement yield (i.e., diagnostic accuracy) was extremely high and also homogeneous among the different centers, without registering any particular difficulty of obtaining a safe scope position or performing a fanning biopsy.

In the multivariate analysis, the diagnostic accuracy was strongly related to the age of the patients and to vascular invasion. These data are reasonable because the presence of more advanced malignant lesions is more frequent in the elderly population and the feasibility and the chance of technical success of the sampling procedure are higher for larger lesions (i.e., those with more frequent vascular involvement).

This study had several limitations. Its retrospective design may be the most important limitation. The databases of each single center have been prospectively collected; however, a selection bias cannot be excluded considering that different needles with various shapes and sizes are available in each center and could have been used in selected patients as alternatives to the study needles. Moreover, the lack of centralized pathologic reading could have caused measurement bias in the histologic evaluation even if all pathologists were specialized in pancreatico-biliary disease. A third limitation is the differing contribution of the centers to the study population, with three centers providing more than half of the total patients.

However, our study also has considerably high strength. The reference diagnosis of malignant or benign lesions was based on a very long (at least 12 months) follow-up period, which makes our results highly reliable. This monitoring period is sufficient to recognize malignant neoplasms in patients with suspicious ultrasound/radiologic findings without evidence of malignancy at biopsy. The results from the different centers were very homogeneous, independently of the number of enrolled patients, which is likely to minimize the effect of a potential selection bias. Among the eight Italian centers that participated in the study, only four were high-volume centers; however, all endosonographers were experts with at least 5 years of operative EUS practice. This means that our data are likely to be reproducible in real life, provided that EUS-FNA is performed by expert endoscopists and expert pathologists.

In conclusion, our data suggest that $19-\mathrm{G}$ flexible needles can be used safely through the duodenum with fewer passes and less time consumed, providing sufficient tissue samples to reach a diagnosis and perform ancillary techniques in most patients. As new 19-G core needles, such as the Franseen and fork-tip needles, have been recently introduced, it would be interesting to compare them with the 19-G flexible needles in terms of feasibility and accuracy when used in a critical position such as in the transduodenal approach.

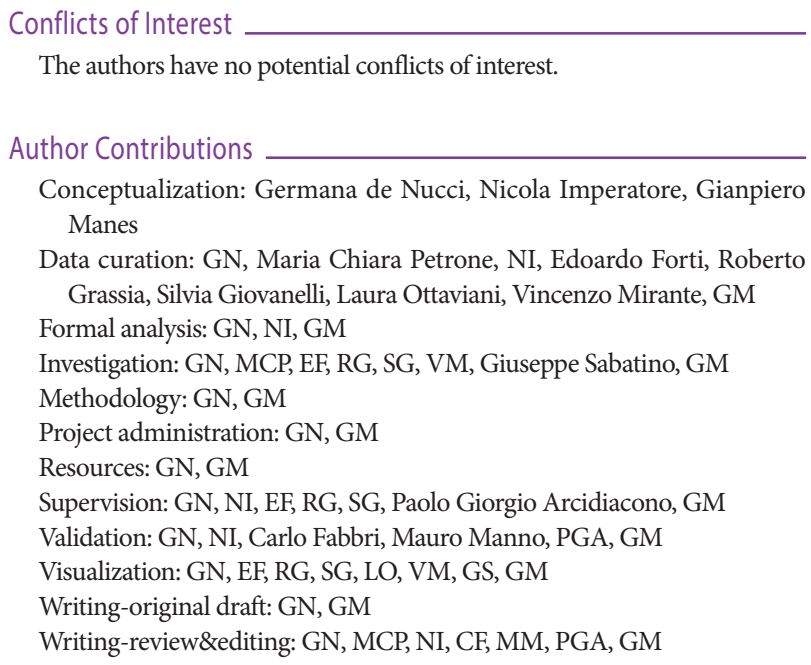

Germana de Nucci: Maria Chiara Petrone: Nicola Imperatore: Edoardo Forti: Roberto Grassia: Silvia Giovanelli: Laura Ottaviani: Vincenzo Mirante: Giuseppe Sabatino: Carlo Fabbri: Mauro Manno: Paolo Giorgio Arcidiacono: Gianpiero Manes:
https://orcid.org/0000-0001-8758-654X https://orcid.org/0000-0002-1045-209X https://orcid.org/0000-0003-3230-6832 https://orcid.org/0000-0002-7400-2979 https://orcid.org/0000-0003-4491-4050 https://orcid.org/0000-0003-3766-9018 https://orcid.org/0000-0003-4791-6609 https://orcid.org/0000-0002-2924-4717 https://orcid.org/0000-0002-1613-6792 https://orcid.org/0000-0003-0670-9910 https://orcid.org/0000-0002-7854-1080 https://orcid.org/0000-0001-6692-7720 https://orcid.org/0000-0002-5319-588X

\section{REFERENCES}

1. Polkowski M, Jenssen C, Kaye P, et al. Technical aspects of endoscopic ultrasound (EUS)-guided sampling in gastroenterology: European So- 
ciety of Gastrointestinal Endoscopy (ESGE) technical guideline - March 2017. Endoscopy 2017;49:989-1006.

2. DeWitt J, Cho CM, Lin J, et al. Comparison of EUS-guided tissue acquisition using two different 19-gauge core biopsy needles: a multicenter, prospective, randomized, and blinded study. Endosc Int Open 2015;3:E471-E478.

3. Thomas T, Kaye PV, Ragunath K, Aithal G. Efficacy, safety, and predictive factors for a positive yield of EUS-guided Trucut biopsy: a large tertiary referral center experience. Am J Gastroenterol 2009;104:584-591.

4. Larghi A, Verna EC, Stavropoulos SN, Rotterdam H, Lightdale CJ, Stevens PD. EUS-guided trucut needle biopsies in patients with solid pancreatic masses: a prospective study. Gastrointest Endosc 2004;59:185190.

5. Iglesias-García J, Abdulkader I, Lariño-Noia J, Domínguez-Muñoz JE. Evaluation of the adequacy and diagnostic accuracy of the histology samples obtained with a newly designed 19-gauge EUS histology needle. Rev Esp Enferm Dig 2014;106:6-14.

6. Shah JN, Ahmad NA, Beilstein MC, Ginsberg GG, Kochman ML. Clinical impact of endoscopic ultrasonography on the management of malignancies. Clin Gastroenterol Hepatol 2004;2:1069-1073.

7. Levy MJ, Jondal ML, Clain J, Wiersema MJ. Preliminary experience with an EUS-guided trucut biopsy needle compared with EUS-guided FNA. Gastrointest Endosc 2003;57:101-106.

8. Varadarajulu S, Hasan MK, Bang JY, Hebert-Magee S, Hawes RH. Endoscopic ultrasound-guided tissue acquisition. Dig Endosc 2014;26(Suppl 1):62-69.

9. Bang JY, Varadarajulu S. Procore and flexible 19 gauge needle can replace trucut biopsy needle? Clin Endosc 2013;46:503-505.

10. Varadarajulu S, Bang JY, Hebert-Magee S. Assessment of the technical performance of the flexible 19-gauge EUS-FNA needle. Gastrointest Endosc 2012;76:336-343.

11. Varadarajulu S, Blakely J, Latif SU, Eloubeidi MA. Quality assessment of current EUS-FNA assembly performance: adequate for use or opportunity for improvement? Gastrointest Endosc 2011;73(4 Sup-
pl):AB174-AB175.

12. Itoi T, Itokawa F, Sofuni A, et al. Evaluation of 19-gauge endoscopic ultrasonography aspiration needles using various echoendoscopes. Endosc Int Open 2013;1:24-30.

13. Attili F, Fabbri C, Yasuda I, et al. Low diagnostic yield of transduodenal endoscopic ultrasound-guided fine needle biopsy using the 19-gauge Flex needle: a large multicenter prospective study. Endosc Ultrasound 2017;6:402-408.

14. Laquière A, Lefort C, Maire F, et al. 19 G nitinol needle versus $22 \mathrm{G}$ needle for transduodenal endoscopic ultrasound-guided sampling of pancreatic solid masses: a randomized study. Endoscopy 2019;51:436443.

15. Itoi T, Itokawa F, Sofuni A, et al. Puncture of solid pancreatic tumors guided by endoscopic ultrasonography: a pilot study series comparing Trucut and 19-gauge and 22-gauge aspiration needles. Endoscopy 2005;37:362-366

16. Panic N, Larghi A. Techniques for endoscopic ultrasound-guided fine-needle biopsy. Gastrointest Endosc Clin N Am 2014;24:83-107.

17. Kopelman Y, Marmor S, Ashkenazi I, Fireman Z. Value of EUS-FNA cytological preparations compared with cell block sections in the diagnosis of pancreatic solid tumours. Cytopathology 2011;22:174-178.

18. Yasuda I, Tsurumi H, Omar S, et al. Endoscopic ultrasound-guided fine-needle aspiration biopsy for lymphadenopathy of unknown origin. Endoscopy 2006;38:919-924.

19. Larghi A, Verna EC, Ricci R, et al. EUS-guided fine-needle tissue acquisition by using a 19-gauge needle in a selected patient population: a prospective study. Gastrointest Endosc 2011;74:504-510.

20. Varadarajulu S, Wilcox CM, Tamhane A, Eloubeidi MA, Blakely J, Canon CL. Role of EUS in drainage of peripancreatic fluid collections not amenable for endoscopic transmural drainage. Gastrointest Endosc 2007;66:1107-1119.

21. Kumbhari V, Peñas I, Tieu AH, et al. Interventional EUS using a flexible 19-gauge needle: an international multicenter experience in 162 patients. Dig Dis Sci 2016;61:3552-3559. 